\title{
Eighty-Seven Consecutive Sleeve Gastrectomies Between the Two Peaks of the Covid-19 Pandemic. An Opening-Phase Experience
}

\author{
Mehmet Ali Yerdel $^{1}$ (D) $\cdot$ Görkem Özgen ${ }^{1}$
}

Received: 22 December 2020 / Revised: 27 February 2021 / Accepted: 4 March 2021 / Published online: 11 March 2021

(C) The Author(s), under exclusive licence to Springer Science+Business Media, LLC, part of Springer Nature 2021

\begin{abstract}
Purpose During the Covid-19 pandemic, the outcome of symptomatic Covid-19 infection occurring early after elective operations is reportedly associated with fatalities. Incidence is unknown and data on bariatric practice is scarce. Covid-19 exposure status and outcomes of sleeve gastrectomy (SG) between the first two peaks of the pandemic are prospectively evaluated.

Material and Methods During our "opening-phase," candidates for SG were enrolled after written informed consent was obtained which specifically emphasized the additional risks of the Covid-19. Viral exposure history and swab/RNA testing were obtained from all. Preoperative antibody testing was also performed, once became available. Preoperative workout, definitions, and surgical technique were standard. Patients were followed up with video-calls. All perioperative data is prospectively recorded.

Results Between June 23 and November 20,87 consecutive SGs were performed without mortality and conversion with a $1.2 \%$ major early complication rate. Single complication was due to Covid-19, acutely becoming symptomatic one day following the SG. During the first year of the pandemic, a minimum of $13.8 \%$ of the patients had encountered the virus and the rate of developing postoperative symptomatic Covid-19 was $6.3 \%$ including a patient with full-blown Covid-19 pneumonia 1 day after SG. Results on weight loss matched expectations.

Conclusion Currently, differing from the first peak of pandemic, vaccines are underway although a more serious surge continues. Given the high rate of morbidity and mortality of Covid-19 infection early after elective operations, caution is warranted when balancing the expected benefit from an elective procedure against the risk of acquiring perioperative Covid-19 infection.
\end{abstract}

Keywords Covid- $19 \cdot$ sleeve gastrectomy $\cdot$ SARS-CoV-2 $\cdot$ complication $\cdot$ elective surgery

\section{Introduction}

During the initial surge of Covid-19, surgeons in affected areas were forced to postpone elective procedures. Once the number of infections declined, an "opening-phase" for elective surgery followed. At that time, no specific therapy or vaccination for Covid-19 was available.

Developing symptomatic Covid-19 after elective operations in the early postoperative period has serious, potentially fatal consequences [1-3]. The morbidity after gastric bypass [4] and sleeve gastrectomy (SG) [5] has already been documented. Incidence remains unknown. No information, except

Mehmet Ali Yerdel

yerdel@yerdel.com

1 İstanbul Bariatrics, Obesity and Advanced Laparoscopy Center, Fulya Mah. Yeșilçimen Sok. 12/407 34394, Şişli, İstanbul, Turkey for a recent survey [6] and case reports $[4,5]$, is available regarding the outcome of bariatric procedures during the pandemic. Many parts of the world are experiencing a second and even worse surge of infections at present. However, unlike the initial phase, vaccination [7] brings hope for a relatively quick solution. Re-assessment of elective and semi-urgent criteria for each surgical discipline may be required, until large-scale vaccination brings Covid-19 under control.

The presented series of 87 consecutive sleeve gastrectomy (SG) operations performed between the first two peaks of Covid-19 pandemic can provide some insight. This is the largest single-center data dealing with Covid-19 in consecutive SGs during the first year of the pandemic. 


\section{Material and Methods}

All procedures performed on patients were in accordance with the ethical standards of the institutional and/or national research committee and with the 1964 Helsinki declaration and its later amendments or comparable ethical standards. The study was approved by the institutional ethics committee and was conducted at a private bariatric center and hospital in İstanbul.

National and Regional Covid-19 Status The $1^{\text {st }}$ official Covid19 case in Turkey was announced on March 11. Our center deployed a lockdown strategy except for emergencies on March 14 (Fig. 1). Similar to what was observed in Italy [8], the vast majority of non-oncological elective procedures in Turkey were postponed in line with the ban imposed by our Ministry of Health on March 20, when the hospital was also officially declared as a pandemic hospital. The national lockdown and the ban for elective procedures were called off on June 3. There were no hospitalized Covid-19 patients when we restarted our program on June 23. During this "openingphase," except for a few unintentional exceptions as in one of our cases, no Covid-19 patient was knowingly admitted to the hospital until the second surge in November, when admissions to the hospital and to ICU started to increase quickly. During periods when there were Covid-19 patients in the hospital, we were able to provide Covid-19-free pathways, allowing separation of bariatric cases from the Covid-19 patients in terms of wards, surgical theaters, and ICU rooms. Soon before the second peak, due to increasing hospital/ICU Covid-19 admissions, we reclosed our elective bariatric practice on November 20.

National Treatment Protocol for Covid-19 In Turkey, all symptomatic Covid-19 patients are treated orally with favipiravir (Favira ${ }^{\circledR}, 200 \mathrm{mg}$, Novelfarma, Turkey) which is provided free of charge by the government. Favipiravir [9] is a viral polymerase and protease inhibitor like remdesivir, and it is the only antiviral agent approved for the treatment of Covid19 in our country.

All candidates were specifically informed about the risks due to the Covid-19 pandemic, and informed consent was obtained from all individual participants included in the study. In obtaining written informed consent, the potential for the virus to influence the outcome of SG and the uncertainties in the world literature were discussed in detail. Viral exposure

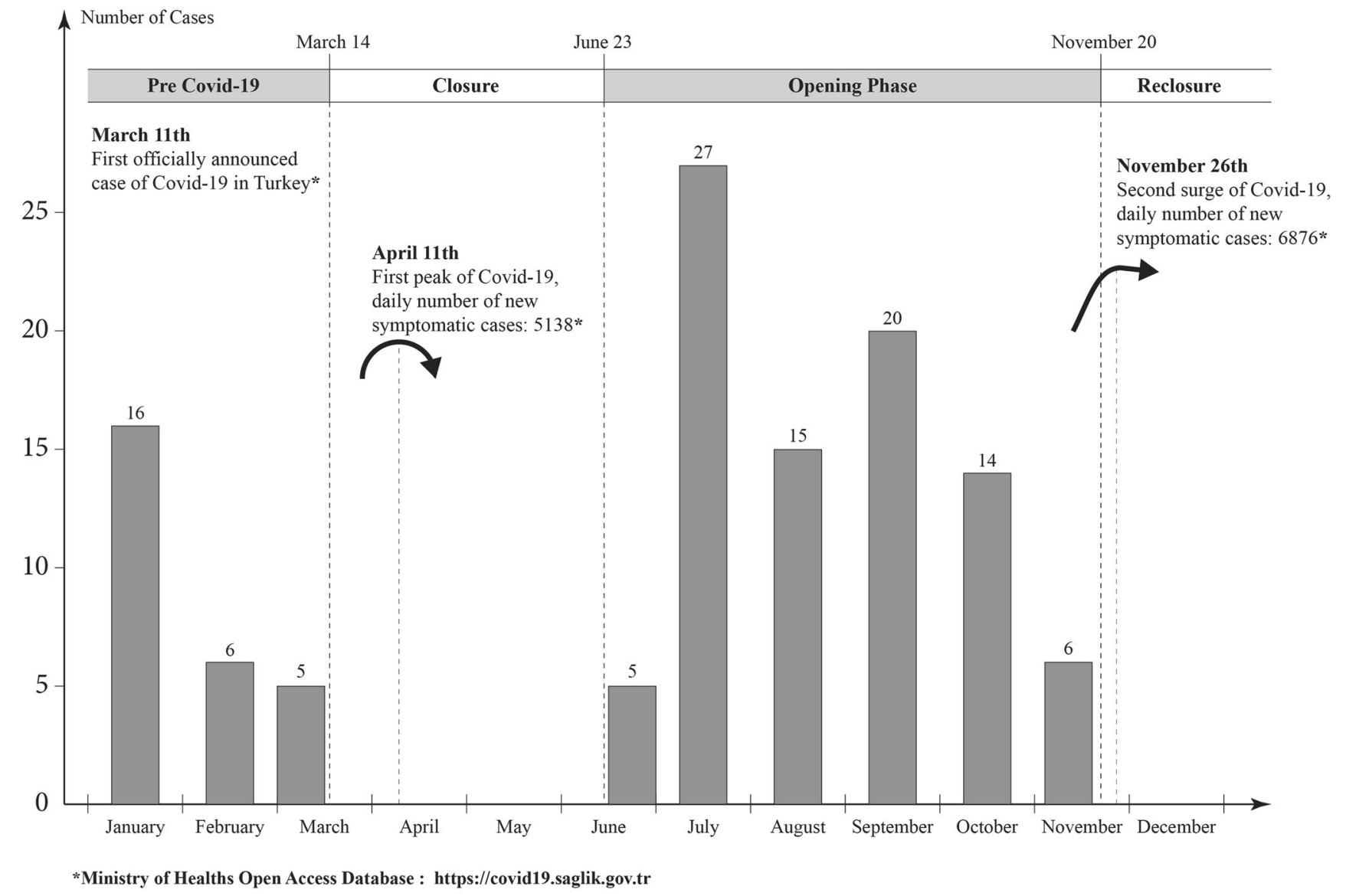

Fig. 1 The timeline of Covid-19 pandemic phases and sleeve gastrectomies 
history was taken. All patients underwent a swab test using polymerase chain reaction (PCR) (Bioeksen, Turkey) 2-7 days prior to the operation and were asked to quarantine until the operation. Eighty-seven percent of the PCRs were done between 2 and 5 preoperative days. Total antibodies (SARSCoV-2 Total, Siemens, Germany) and IgG/IgM + IgA (ELISA, Vircell, Spain) were also studied, once kits became available.

Preoperative Workout All patients were evaluated by a team comprising surgeon, dietician, psychologist, psychiatrist, cardiologist, pulmonologist, endocrinologist, and anesthetist. Blood analysis, gastroscopy, abdominal ultrasound, chest radiograph, echocardiography, and lung function tests were routine. All patients were trained to adopt a low-calorie diet 1014 days before surgery. During the preoperative preparation and hospitalization, all patients were asked to use double masks, and one of our team members helped train them to avoid any breach regarding distancing or mask usage.

Thromboprophylaxis For the prevention of venous thromboembolism, all patients received enoxaparin sodium (Clexane, pre-filled syringe, Sanofi, Turkey) by preoperative subcutaneous injection according to body mass index (BMI, $\mathrm{kg} / \mathrm{m}^{2}$ ) (BMI $>50$ received $60 \mathrm{mg}$, BMI $<50$ received $40 \mathrm{mg}$, repeated every $24 \mathrm{~h}$, for 10 days). Patients who were already on oral or novel anti-coagulants were managed by bridging with enoxaparin and weaning off other anti-coagulation. Pneumatic stocking usage during surgery/hospitalization and early mobilization was routine.

SG Technique Using an optical trocar for first entry, with a 5trocar technique, an SG over a 42F bougie, starting 3-4 cm away from the pylorus, was performed. The staple line was routinely reinforced by continuous stitching with a barbed suture (V-Loc 180; Medtronic, Minneapolis, MN, USA). A low pneumoperitoneum protocol was applied to all, and no filters were used. Once the trocars were in place, the pneumoperitoneum was set to $8-10 \mathrm{mmHg}$ and increased if necessary. Operating room personnel were kept to a minimum. Including the nurse, the scrub team was limited to three. Senior surgeon performed all SGs (M.A.Y.). All wore 2 masks and a shield, one mask being an $\mathrm{n} 95$. An intraoperative leak test was used selectively, and a contrast swallow on postoperative day (POD) 1 was routine. All patients were drained with a 7-mm Jackson Pratt drain, which was removed before discharge, when the total drain output was recorded. Unlike our preCovid-19 protocol which dictated routine discharge at third postoperative day, early discharge was encouraged.

Unless absolutely necessary, follow-ups were done by daily video calls in the 1 st week and weekly video calls thereafter. Weight loss is defined by $\%$ excess weight loss (\% EWL).

\section{Results}

All patients were tested negative for Covid-19 by PCR preoperatively. Preoperative antibodies were studied in 76 patients. IgG was positive in 7 patients who all had a PCR-proven Covid-19 history, one being asymptomatic and 6 suffered symptomatic disease 1-5 months before. No Covid-19related abnormality was found at preoperative assessment of these 6 patients, but $\operatorname{IgM}+\operatorname{IgA}$ was also positive in 2 . A second PCR was negative in both, and in the absence of any clinical abnormality, SG was not denied. No viral infection was detected during the follow-up of these patients.

The timeline of SGs, in relation to our closure/opening/ reclosure phases and to the 2 peaks of the national Covid-19 epidemic, is presented in Fig. 1. Eighty-seven consecutive SGs were performed without mortality, conversion, or any intra-/postoperative complication, except for one major complication: Covid-19 pneumonia/pancreatitis. In 7 patients, hiatal hernias were repaired by primary closure of the hiatal defect with 00 silk sutures. Perioperative data is summarized in Table 1 . In 47 patients who completed the $3^{\text {rd }}$ month follow-up, \% EWL was $59.4 \pm 23.2$ (range: 28.8-129.8).

Table 1 Perioperative data of 87 patients/sleeve gastrectomies

\begin{tabular}{ll}
\hline Demographics & \\
\hline Body mass index $\left(\mathrm{kg} / \mathrm{m}^{2}\right)$ mean (range) & $38.8 \pm 5.4(28.8-59.9)$ \\
Male/female & $24 / 63$ \\
Age (years) mean (range) & $35.6 \pm 10.3(15-66)$ \\
Comorbidities & \\
Hypertension & $36(41.4)$ \\
Type 2 diabetes mellitus $n(\%)$ & $8(9.2)$ \\
Insulin resistance $n(\%)$ & $66(75.9)$ \\
Metabolic syndrome $n(\%)$ & $66(75.9)$ \\
Covid-19 status & \\
History for Covid-19 $n(\%)$ & $6(6.9)$ \\
Preoperative swab/PCR & Done in $87 /$ all negative \\
Preoperative total antibody & Done in 33/all negative \\
Preoperative IgG/IgM + IgA & Done in 43/see text \\
Pneumoperitoneum & \\
8 mmHg $n(\%)$ & $3(3.5)$ \\
10 mmHg $n(\%)$ & $37(42.5)$ \\
$11-14$ mmHg $n(\%)$ & $47(54)$ \\
Mean operating time minutes (range) & $93 \pm 18.7(63-138)$ \\
Discharge time & \\
2 days $n(\%)$ & $5(5.8)$ \\
3 days $n(\%)$ & $81(93)$ \\
8 days $n(\%)$ & $1(1.2)$ \\
Follow-up $($ days $)$ mean (range) & $98.1(21-162)$ \\
PCR, polymerase chain reaction & \\
\hline & \\
\hline &
\end{tabular}


During the timeline, 12/87 (13.8\%) of the patients were diagnosed with Covid-19 by PCR. Seven of these were preSG, and 5 had symptomatic post-SG disease (6.3\%). Four mildly symptomatic Covid-19 cases were diagnosed on the $3^{\text {rd }}, 4^{\text {th }}$ ( 2 patients), and $5^{\text {th }}$ postoperative months. None required hospital admission, all treated orally with favipiravir without sequelae. In one case, Covid-19 became evident in POD 1 with high fever/pulmonary involvement. This was a 29-year-old male, body mass index of $42.1 \mathrm{~kg} / \mathrm{m}^{2}$ with metabolic syndrome who had been tested negative by PCR 2 days prior to surgery. $\operatorname{IgG}$ and $\operatorname{IgM}+\operatorname{IgA}$ were also negative. His preoperative chest X-ray/echocardiography/respiratory function tests were normal. POD 2 tomography revealed right pulmonary infiltrates with no other sign of a leak, collection, or abdominal abnormality. Cough/fatigue ensued though $\mathrm{sO}_{2}$ level remained $>95 \%$. No abdominal complaints occurred, and oral liquid/protein intake was supplemented with intravenous hydration/nutrition/antibiotics. On POD 5, PCR became positive, and favipiravir was started orally, and enoxaparin sodium dose was increased to $2 \times 60 \mathrm{mg}$ subcutaneously. The patient started to suffer from severe emotional stress due to isolation and left the hospital on POD 8 against our advice. Further hospitalization was felt appropriate due to the presence of pulmonary involvement and the newly diagnosed, asymptomatic acute pancreatitis, which became evident with sequentially increasing lipase/amylase values. No risk factor, besides surgical trauma, was present for pancreatitis. Recent literature [10] showing pancreatitis inducing effect of Covid19 raised suspicion for the virus being the cause of pancreatitis. Contrary to our misgivings, the patient did well at home. Increased calorie/protein liquid oral intake was enforced with daily calls. He completed antibiotic, antiviral (5 days), and enoxaparin ( 25 days) therapies at home. At $10^{\text {th }}$ postoperative day, fever and cough subsided, and except for reduced mobility due to fatigue, he became asymptomatic. Lipase/amylase levels returned to normal on POD 20. He weighs $25 \mathrm{~kg}$ less 2.5 months after surgery, without any sequelae. Presently, he is $\operatorname{IgG}$ positive and PCR negative.

\section{Discussion}

Our "opening-phase" experience between the two peaks of the 2020 pandemic showed that a "minimum" of $13.8 \%$ of the patients were infected with the virus. The "minimum" rate of developing postoperative symptomatic Covid-19 was $6.3 \%$. These rates are "minimum" because the definition of infection is only based on the identification of the viral RNA by PCR, which has a relatively high rate for false negative results. Furthermore, PCR was only performed once with the exception of those with Covid-19 like symptoms. Asymptomatic individuals remained untested postoperatively. Lastly, since we now know that re-infections are possible, those percentages surely can only reflect the minimum rate for viral infections. Nevertheless, the sole major complication was an early postoperative Covid-19 infection, giving a $1.2 \%$ rate for major complications. The results for weight loss matched expectations.

Since obesity is a risk factor for poor outcome/mortality in Covid-19 infection, bariatric surgery, being considered a risk reducing intervention for Covid-19, was widely practiced after the initial surge subsided. Covid-19 pandemic even caused a mind-shift, imparting a semi-urgent status to an otherwise elective weight reduction procedure. Simultaneously, early postoperative Covid-19 infections after elective operations (i.e., cholecystectomy, hernia repair) were shown to be potentially fatal [1], and a striking mortality rate of $20 \%$ was reported in patients undergoing surgery during the incubation period of Covid-19 infection [2]. High mortality related to pulmonary complications in patients undergoing surgery with perioperative Covid-19 infection was the subject of another recent report [3]. In addition, high morbidity and the complexity of management of early postoperative Covid-19 infection after gastric bypass [4] and SG [5] were documented. Concerns regarding the difficulty of treatment of Covid-19 in postbariatric surgery patients related to oral intake/absorption problems have been appropriately raised [11]. The aforementioned mind-shift, therefore, has to be balanced by the risk of acquiring Covid-19 in the early postoperative period which is a reality as shown herein and by others [1-6]. Notably, any benefit to patients in terms of outcome of Covid-19 infection will not become relevant for at least a few months after surgery, until appreciable weight loss has occurred. Surely, no guarantees can be given to avoid Covid-19 infection until then.

POD 1 Covid-19 case demonstrates the fallibility of the nasopharyngeal PCR testing by swabs. Hamilton et al. deserve credit in their effort to develop routine preoperative testing of individuals undergoing planned elective procedures by swab PCRs [12]. They also stressed all the difficulties and the associated logistic hurdles, causing a failure rate of $4 \%$ just to be able to do testing. The exact incidence of false negative tests and the risk of developing early postoperative Covid-19 disease remains unknown.

Until recently, bariatric surgeons had to contend with these potential risks/benefits and some of the limitations in knowledge about Covid-19. Although no specific treatment agent including favipiravir is shown to be clearly effective or superior to another, the most important development, which is likely to have a groundbreaking impact, is the apparent success of several vaccines and approval of these by regulatory authorities. If vaccination is proved effective in large scale, it seems clear that elective bariatric surgery should take place only in immune patients.

Until full vaccine coverage becomes available, new surges and possibly new closure/openings can be expected. Unlike 
many other elective operations, weight reduction surgery is a major fore-gut procedure with an intrinsic serious morbidity rate of around $1 \%$. In addition, it severely affects oral intake, and this is especially true for restrictive procedures such as SG, the most commonly performed primary bariatric procedure worldwide. Both the patients and the medical personnel must be fully aware of the possibility of developing severe Covid-19 infection during the early postoperative period. Surgeons must be more cautious when balancing the expected benefit from an elective procedure against the risk of acquiring perioperative Covid-19 infection. Considering bariatric surgery semi-urgent may be the wrong approach, especially since there is now genuine hope that the pandemic will be over soon.

Acknowledgements Authors want to thank to Murat Akyol M.D., F.R.C.S., for reviewing the article and for his linguistic support.

\section{Declarations}

Conflict of Interest The authors declare no competing interests.

\section{References}

1. Aminian A, Safari S, Razeghian-Jahromi A, et al. COVID-19 outbreak and surgical practice. Unexpected fatality in perioperative period. Ann Surg. 2020;272:e27-9.

2. Lei S, Jiang F, Su W, et al. Clinical characteristics and outcomes of patients undergoing surgeries during the incubation period of COVID-19 infection. E Clin Med. 2020;
3. COVID Surg Collaborative. Mortality and pulmonary complications in patients undergoing surgery with perioperative SARS CoV-2 infection: an international cohort study. Lancet. 2020;396: 27-38.

4. Aminian A, Kermansaravi M, Azizi S, et al. Bariatric surgical practice during the initial phase of COVID-19 outbreak. Obes Surg. 2020;30:3624-7.

5. Taube T, Mansour S, Hakky S. A Unique presentation of COVID19 in a patient post sleeve gastrectomy. Obes Surg. 2020;30:35889.

6. Shinghai R, Tahrani AA, Ludwig C, et al. Global 30-day outcomes after bariatric surgery during the COVID-19 pandemic (GENEVA): an international cohort study. Lancet Diabetes Endocrinol. 2021;9:7-9.

7. Walsh EE, Frenck RW Jr, Falsey AR, Kitchin N, Absalon J, Gurtman A et al. Safety and immunogenicity of two RNA-based Covid-19 vaccine candidates. N Engl J Med. 2020.

8. Marinari GM, Anselmino M, Tascini C, et al. Bariatric and metabolic surgery during COVID-19 outbreak phase 2 in Italy: why, when and how to restart. Surg Obes Relat Dis. 2020;16:1614-8.

9. Chen PJ, Chao JM, Lai CC. Clinical efficacy and safety of favipravir in the treatment of Covid-19 patients. J Infect. 2020;S0163-4453(20):30766-0. https://doi.org/10.1016/j.jinf. 2020.12.005.

10. Goyal H, Sachdeva S, Perisetti A, et al. Hyperlipasemia and potential pancreatic injury patterns in COVID-19: A marker of severity or innocent bystander? Gastroenterology; 2020.

11. Azran C, Porat D, Dahan A, et al. Treatment of COVID-19 Patients Post-Bariatric Surgery: Issues for Consideration. J Clin Med. 2020;9:2827.

12. Hamilton BSC, Kratz JR, Sosa JA, et al. Developing perioperative Covid-19 testing protocols to restore surgical services. NEJM Catalyst. 2020;

Publisher's Note Springer Nature remains neutral with regard to jurisdictional claims in published maps and institutional affiliations. 